\title{
Is an Ancient Genome Repair Mechanism the Trojan Horse of Cancer?
}

\author{
Vladimir F Niculescu*
}

Cell biologist, Germany

ISSN: 2637-773X

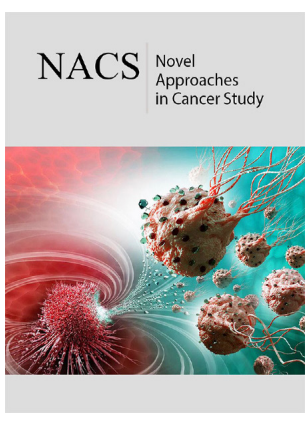

*Corresponding author: Vladimir F Niculescu, Cell biologist, Kirschenweg 1, 86420 Diedorf, Germany

Submission: 㙁June 04, 2021

Published: 制June 14, 2021

Volume 5 - Issue 5

How to cite this article: Vladimir F Niculescu. Is an Ancient Genome Repair Mechanism the Trojan Horse of Cancer?. Nov Appro in Can Study. 5(5). NACS.000625. 2021. DOI: $10.31031 /$ NACS.2021.05.000625

Copyright@ Vladimir F Niculescu, This article is distributed under the terms of the Creative Commons Attribution 4.0 International License, which permits unrestricted use and redistribution provided that the original author and source are credited.
Keywords: Cancer; Germline; Cancer germ stem cells; Genome repair; PGCC; EMT; MET

Abbreviations: aCLS: Autonomous Cyst-Like Structure; CAM: Cell Agglutinating Molecules; DSB: Double Strand Break; GSC: Germ Stem Cells; MGRS: Multinucleated Genome Repair Structure; PGCC: Polyploid Giant Cancer Cell; SGT: Soma-To-Germ Transition

\section{Introduction}

In the last decade, more and more researchers believe that cancer is caused by a progressive weakening of a multicellular cell, leading to a loss of function and a switch to a unicellular life cycle. Lineweaver et al. [1] consider cancer as "a dysregulation and degeneration of recently evolved genes and the upregulation of complementary ancient genes." This also applies to DNA repair genes. Multicellular cells have differentially dysfunctional DNA repair systems [2]; second, more evolved repair genes (evolutionary younger genes) become dysfunctional more rapidly than older genes. Also of particular interest is the consideration by Lineweaver et al. [1] that cancer "can only revert to phenotypes compatible with cellular survival in a human" [3]. This fits with the analogy described between the dual cell systems of cancer and intestinal parasitic amoebae [4-9].

If the scenario postulated by Lineweaver et al. [1] holds, the weakening cell-oforigin of cancer or even its progenitors must rely on older repair mechanisms, e.g., from unicellular ancestors, although such repair pathways are not particularly suited to repair multicellular genomes. Ancient repair mechanisms are known from intestinal parasitic amoebae (Entamoeba) [10]. Amoebae can remove deleterious DNA lesions from the oxygensensitive germline cells damaged by hyperoxia (excess oxygen). Defective cells fuse to form Multinucleated Genome Repair Syncytia (MGRS) [10]. Within the MGRS, genome repair occurs through polyploidization, nuclear fusion, and accumulation of the defective genome copies into a giant MGRS nucleus.

The giant nucleus repairs and reorganizes the fused chromatin into fully functional genome copies and distributes them into spore-like germ stem cells. Ancient MGRS mechanisms are conserved in the dark genome of mammalians. In cancer, they are reactivated, not only at cancer initiation, but each time the cancer germline loses its reproductive function through stress.

However, the reactivated MGRS pathway is a tool of the unicellular life and has a dramatic effect on the weakened multicellular cell that reactivates it. The price paid by the weakened cell and its progeny is high: they radically change their way of life (cell fate) and follow the rules of the unicellular germ and soma cell systems. [7,9]. In my opinion, the reactivated ancient genome repair mechanism is the Trojan horse causing the oncogenic transformation.

\section{Ambient Oxygen Controlls Germline's Cell Fate}

The recent germ and soma theory of cancer of unicellular imprinting [9] explains the relationship between the germline transition from asymmetric to symmetric cell cycles, the 
loss of reproductive function, and the accumulation of MRGSs in late stages of cancer and recurrence. All these events are due to better oxygenation of later cancers and tumors.

Depending on the ambient oxygen, the unicellular germ and soma cell system of cancer proliferates either by symmetric or by asymmetric cell cycles. Oxygen levels below 5.7\% 02 (normoxia, physoxia) favor asymmetric cell division, whereas oxygen levels above $5.7 \%$ (hyperoxia) switch cancer cells to symmetric cell cycles. Germ and soma respond differently to oxygen: while the germline is an oxygen-sensitive cell line, soma cells are oxygentolerant. Depending on the oxygen content of the environment, the germline proliferates with or without the production of Germ Stem Cells (GSCs) [9].

Normoxia favors and maintains the reproductive state of the germline and the formation of GSCs. Under physoxic conditions, the germline differentiates self-renewing progenitor cells and committed precursor cells for reproductive- amitotic cell cycles (polyploid aCLS cycles) [9]. Cells that perform the reproductive aCLS cycle increase their genome copies number through subsequent rounds of endoreplication and generate daughter GSCs, which in turn differentiate germ and soma. Normoxia (physoxia) favors and maintains the reproductive state of the germline.

Hyperoxia converts the reproductive germline to a nonreproductive state that proliferate by symmetric cell division (two identical daughter cells) without differentiation into self-renewing progenitor and committed precursor cells. DNA (DSB) lesions caused by excess oxygen do not allow reproductive aCLS cycles, However, cancer cell populations require full functional germlines. further GSC accumulation and reproductive germlines. To recover reproductive germlines and clones the cancer cell system uses, on the one hand, Soma-To-Germ Transition (SGT) and the other hand the repair and reorganization genome pathway through MGRSs [10].

\section{MGRSs in Early and Later Stages of Cancer}

MGRSs are better known in the cancer literature as "preexisting Polyploid Giant Cancer Cells (PGCCs)"and are frequently observed in untreated cancers [11]. when damaged germline cells lose reproductive function and the ability to form GSCs, requiring germline renewal through genome reorganization. This occurs frequently at later cancer stages and recurrence, and least frequently at early cancer stages when undamaged germline cells continuously differentiate GSCs through aCLS cycles analogous to protists [10]. GSCs differentiate in turn further germ and soma [9].

Late cancer stages show not only the accumulation of MGRS but also the switch from asymmetric to symmetric cell division $[12,13]$. Nevertheless, the correlation between these two events was not previously understood. By analogy with the evolution of the germ and soma systems of protist, the germ and soma theory of cancer [9] has recently been explaining not only the switch from asymmetric to symmetric cell division at later stages of cancer but also the transition from the reproductive to the non-reproductive germline state (no GSC production) and the repair and reorganization pathway by MRGSs. All of these processes occur at later stages due to the better oxygenation of later cancers and tumors. While the "less oxygenated" zones and niches of early cancers favor precursor cell differentiation and GSC production, the "better oxygenated" zones of later cancers irreversible damage the germline and the clones generated by SGT events. The damaged germline must be repaired and reorganized again and again through the MGRS pathway [9].

\section{Genotoxic Treatments also Inactivate Repair Genes of Younger Evolutionary Age}

PGCCs induced by genotoxic treatments are not identical to the "preexisting" PGCCs (fusion MGRSs) that occur before treatments. Many chemotherapeutic agents inhibit the cell fusion ability expressed in oxygenically damaged germline cells. Only a few genotoxically damaged germline cells such as glioblastoma germline cells, retain the ability to form MGRSs by cell fusion $[9,14]$. When chemotherapeutic agents have damaged genes that controls cell agglutinating molecule (CAM) production and cell fusion ability genotoxically damaged germline cells can activate unicellular repair mechanisms such as hyperploidization and form hyperploid genome repair structures (HGRSs) by endoreplication $[9,10,15]$. Hyperploidization up to 20-40C DNA has also been observed in protists that cycle and divide symmetrically under hyperoxia conditions [9].

The GSCs generated by HGRS can develop germline clones capable to perform reproductive aCLS cycles as observed in MDAMB-231 breast cancer cell culture after exposure to doxorubicin ("second wave of polyploidization") [15]. In other treated cancers, chemotherapeutic agents have more drastic effects and damage the evolutionary young repair genes that control cell fusion and hyperploidization. The drastically damaged germline cells seek evolutionarily older repair genes from the dark genome and laborious repair mechanisms coupled to G2 tetraploidy [TGRS], which often lead to aneuploidy.

\section{The Evolutionary History of the Germ and Soma Cell System}

Cancer is an archaic disease that utilized an ancient germ and soma cell system developed by unicellular ancestors, a long time before the transition to multicellular organism begins. The dual germ and soma cell system of unicellular ancestors has been conserved in the genome of all unicellular and multicellular eukaryotes. Nevertheless, the relationship between the germ and soma cell system of cancers and pathogenic amoebae is incomparably higher than to the primitive germ layers developed by Cidarians and Sponges, as well as all other Metazoans, Vertebrates and Mammalians respectively, their embryonic structures and germ layers.

From an evolutionary perspective, the germline of the germ and soma cell systems is the oldest cell line of these cell systems. The preceding cell line, which later became germline, proliferated at that time under nutrient-poor conditions and low oxygen levels 
and performed asymmetric cell division. Due to scarce and unstable living conditions, one of both non-identical daughter cells stopped proliferating immediately after cell division, forming a resistant reproductive cyst. Harmful environmental conditions, especially increasing oxygen levels, lead to DNA DSB lesions in this oxygen sensitive cell line and damaged the genome. DNA DSB lesions led to loss-of-function and the defective cells lose their ability to form cysts. However, they become fusible and fused into MGRSs. Unfortunately, MGRSs were also damaged in these ancient times by UV radiation.

As oxygen levels increased ( $>5.5 \% 02$ content), the ancient cell line transformed into a true germline to produce Germ Stem Cells (GSCs) capable of dual differentiation. These GSCs were able to differentiate in parallel a second cell line best adapted to the more oxygenated environment. The younger oxygen tolerant cell line worked as a vegetative cell line without reproductive function. It was unable to form cysts. However, it learned to receive deleterious genome signals from germline cells and to respond to such signals with Soma-To-Germ Transformation (SGT). The intraconverted cells develop new reproductive germlines and clones.

\section{References}

1. Lineweaver CH, Davies PC, Vincent MD (2014) Targeting cancer's weaknesses (not its strengths): Therapeutic strategies suggested by the atavistic model. Bioessays 36(9): 827-835.

2. Helleday T, Petermann E, Lundin C, Hodgson B, Ricky S, et al. (2008) DNA repair pathways as targets for cancer therapy. Nat Rev Cancer 8(3): 193204.

3. Lineweaver CH, Bussey KJ, Blackburn AC, Davies PCW (2021) Cancer progression as a sequence of atavistic reversions. BioEssays, 2000305.

4. Niculescu VF (2018) The cancer stem cell family: Atavistic origin and tumorigenic development. MOJ Tumor Res 1(2): 70-73.
5. Niculescu VF (2018) Molecular and cell biological considerations in the initiation and development of sporadic non-hereditary solid cancers. J Cancer Genetics and Biomerkers 1(2): 24.

6. Niculescu VF (2019) The reproductive life cycle of cancer: Hypotheses of cell of origin, TP53 drivers and stem cell conversions in the light of the atavistic cancer cell theory. Medical Hypotheses 123: 19-23.

7. Niculescu VF (2019) aCLS cancers: Genomic and epigenetic changes transform the cell of origin of cancer into a tumorigenic pathogen of unicellular organization and lifestyle. Gene 726: 144174.

8. Niculescu VF (2019) Cancer stem cells and the unicellular life cycle of cancer. Novel Approaches in Cancer Study 3(3): 280-281.

9. Niculescu VF (2021) Germline evolution in cancer as explained by the germ and soma theory of dual cell systems. J Clin Anat Pathol 6(1): 113.

10. Niculescu VF (2021) Have protists and cancer analogous germ and soma cell systems? New insights into the life cycle of Entamoeba histolytica and E. invadens.

11. Zhang S, Mercado-Uribe I, Xing Z, Sun B, Kuang J, et al. (2014) Generation of cancer stem-like cells through the formation of polyploid giant cancer cells. Oncogene 33(1): 116-128.

12. Capp JP (2019) Cancer stem cells: From historical roots to a new perspective. Hindawi Journal of Oncology. Article ID 5189232.

13. Liu C, Zhan Ma Z, Zhen Cai Z, Fengyu Zhang F, Cheng Liu C, et al. (2020) Identification of primordial germ cell-like cells as liver metastasis initiating cells in mouse tumour models. Cell Discovery 6:15.

14. Kaur E, Rajendra J, Jadhav S, Shridhar E, Sastri Goda J, et al. (2015) Radiation-induced homotypic cell fusions of innately resistant glioblastoma cells mediate their sustained survival and recurrence. Carcinogenesis 36(6): 685-695.

15. Salmina K, Bojko A, Inashkina I, Staniak K, Dudkowska M, et al. (2020) "Mitotic slippage" and extranuclear DNA in cancer chemoresistance: A focus on telomeres. Int J Mol Sci 21(8): 2779. 